\title{
Towards Teaching Metacognition: Supporting Spontaneous Self-Assessment
}

\author{
Ido Roll, Eunjeong Ryu, Jonathan Sewall, Brett Leber, Bruce M. McLaren, \\ Vincent Aleven, and Kenneth R. Koedinger \\ Human Computer Interaction Institute, \\ Carnegie Mellon University \\ 5000 Forbes Ave., Pittsburgh PA 15213 \\ \{iroll, eryu\} a andrew. cmu.edu, \\ \{sewall, bleber, mclaren, aleven, koedinger\}@cs.cmu.edu
}

\begin{abstract}
The Self-Assessment Tutor (SAT) is an add-on component to Cognitive Tutors that supports self-assessment in four steps: prediction, attempt, reflection, and projection. The SAT encourages students to self-assess their ability spontaneously while problem solving, and to use help resources accordingly. For that reason its episodes precede the students' work with the Cognitive Tutor, which itself remains unchanged. The SAT offers detailed feedback and help function to support the Self-Assessment process. A complementary instruction is given to students before working with the SAT. We hypothesize that working with the SAT will encourage students to self-assess on subsequent problems requiring similar skills, and thus will promote learning. A classroom evaluation of SAT is currently in progress.
\end{abstract}

\section{Introduction}

Supporting students' metacognition while working with Intelligent Tutoring Systems contributes to deep, meaningful learning of the relevant domain knowledge $[1 ; 2]$ and can promote future learning in various domains and learning environments by equipping students with better metacognitive skills [6;7].

One such skill is self-assessment, i.e., the ability and tendency of students to evaluate correctly their knowledge level. Self-assessment can be used by students to choose their actions and monitor their progress [3], and by the tutoring system to update its assessment of the student [8]. Research shows, however, that students are not good at self-assessing their knowledge [4].

To address that, and as part of an overall metacognitive suite, Gama [3] prompts students to evaluate their knowledge level before each problem, and to reflect on their assessment once they are done. Zapata et al. [8] allow students to self-assess their knowledge by describing their experience with similar concepts.

\section{The Self-Assessment Tutor (SAT)}

The Self Assessment Tutor (SAT) we describe here was built using the Cognitive Tutors Authoring Tools - an environment for authoring tutors by demonstration [5], 
and is an add-on component to existing Cognitive Tutors. Each unit of the Geometry Cognitive Tutor (e.g. Angles) is composed of sections (e.g. angles between parallel lines) during which students practice a specific set of skills (e.g. sameside interior angles). SAT does not prompt students to self-assess their ability on every individual problem since it is very time consuming, and might become annoying to the students. In addition, since students are being prompt to self-

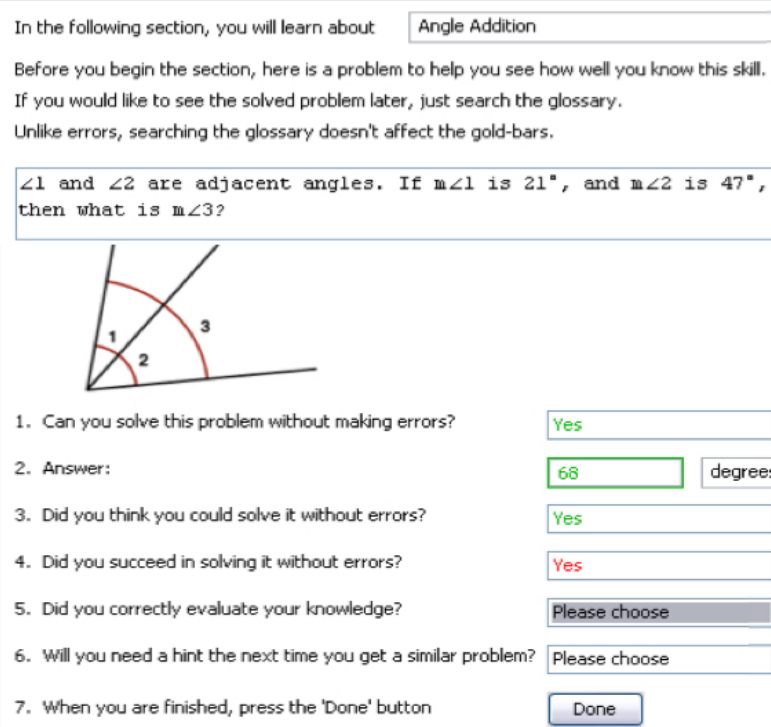

1. Can you solve this problem without making errors?

2. Answer:

3. Did you think you could solve it without errors?

4. Did you succeed in solving it without errors?

5. Did you correctly evaluate your knowledge?

6. Will you need a hint the next time you get a similar problem?

7. When you are finished, press the 'Done' button

Diagram 1. The Self-Assessment Tutor: prediction (q. 1), attempt (q. 2), reflection (q. 3-5) and projection (q. 6) assess, they are not engaged in that behavior spontaneously. To encourage spontaneous self-assessment, SAT adds a self-assessment preparatory activity before each set of problems, and does not interfere with the problem-solving process itself. In practice, between any two sections of the Cognitive Tutor (that remain intact), students engage in a selfassessment episode, in which they assess their ability on the relevant set of skills.

The self-assessment process. Each self-assessment episode includes problems on which students assess their ability - one problem per skill (each episode includes 4-5 such skills). In designing the interface of the SAT we used similar principles to those detailed in Gama [3]. Each problem includes the following steps: (1) Prediction how well do I think I can solve this problem? (2) Attempt - what is the answer to the problem? (3) Reflection - how well did I do? Did it match my prediction? (4) Projection - what does this imply about my ability to solve problems using similar skills in the future? Will I need help the next time I attempt a problem requiring a similar skill?

Each of these steps (besides attempting the problem) is scaffolded with drop-down menus (see diagram 1). Additional support is made available in on-demand hints (not seen in diagram). Using these steps, we try to relate the current self-assessment experience to relevant future situations.

Feedback on self-assessment. Giving feedback on self-assessment should be based on the students' assessment of themselves, not the system's assessment of the student. On this assumption, SAT traces students' attempts and assessment and gives feedback according to the following principles: 
- Where possible, the tutor should base feedback on previous responses (e.g., feedback to question 3, "Did you think you could solve it without errors?" is based on their answer to question 1).

- When the student reports a need for help, it should be given. The system does not assume that the student knows unless the student reports so.

- When several answers are possible, the tutor should allow for all of them.

Self-assessment instruction. Before working with the SAT, each student receives an instruction through a movie, describing the importance of self-assessment and demonstrating the interface of the new tutor.

The SAT is currently being evaluated in a classroom study, and is well received by the students. We hypothesize that it will contribute to learning since students would be more aware of their knowledge level, and would be engaged in self-assessment spontaneously more often.

Acknowledgements. We express thanks to Ido Jamar, Jo Bodnar, Mari Murphy, Sabine Lynn, Kris Hobaugh and Dale Walters for their help carrying out this study.

\section{References}

1. Aleven, V., \& Koedinger, K.R. (2002). An effective metacognitive strategy: learning by doing and explaining with a computer-based Cognitive Tutor. Cognitive Science 26(2), 147-79.

2. Bunt, A., Conati, C., \& Muldner, K. (2004) Scaffolding self-explanation to improve learning in exploratory learning environments. in proceedings of the $7^{\text {th }}$ International Conference on Intelligent Tutoring Systems, 656-67. Berlin: Springer-Verlag.

3. Gama, C. (2004) Metacognition in Interactive Learning Environments: The Reflection Assistant Model. in proceedings of the 7th International Conference on Intelligent Tutoring Systems, 668-77. Berlin: Springer-Verlag.

4. Glenberg, A.M., Wilkinson, A.C., \& Epstein, W. (1982). The Illusion of Knowing: Failure in the Self-Assessment of Comprehension. Memory and Cognition 10, 597-602.

5. Koedinger, K.R., Aleven, V., Haffernan, N., McLaren, B.M., \& Hockenberry, M. (2004). Opening the Door to Non-Programmers: Authoring Intelligent Tutor Behavior by Demonstration. in proceedings of the $7^{\text {th }}$ International Conference on Intelligent Tutoring Systems.

6. Roll, I., Aleven, V., McLaren, B.M., Ryu, E., Baker, R.S.J.d., \& Koedinger, K.R. (to appear) The Help Tutor: Does Metacognitive Feedback Improves Students' Help-Seeking Actions, Skills and Learning? to appear in proceedings of The 8th International Conference on Intelligent Tutoring Systems. Berlin: Springer-Verlag.

7. Roll, I., Baker, R.S., Aleven, V., McLaren, B.M., \& Koedinger, K.R. (2005) Modeling Students' Metacognitive Errors in Two Intelligent Tutoring Systems. in proceedings of User Modeling 2005, 379-88. Berlin: Springer-Verlag.

8. Zapata-Rivera, J.D., \& Greer, J.E. (2004). Interacting with Inspectable Bayesian Student Models. Int J of Artificial Intelligence in Education 14(2), 127-63. 